\title{
Effect of material properties on reverse flow in nematic liquid crystal devices with homeotropic alignment
}

\author{
Pieter J. M. Vanbrabant, ${ }^{1, a)}$ Jeroen Beeckman, ${ }^{1}$ Kristiaan Neyts, ${ }^{1}$ Richard James,${ }^{2}$ and \\ F. Anibal Fernandez $z^{2}$ \\ ${ }^{1}$ Department of Electronics and Information Systems, Ghent University, B-9000 Ghent, Belgium \\ ${ }^{2}$ Department of Electronics and Electrical Engineering, University College London, WC1E 7JE London, \\ United Kingdom
}

(Received 20 August 2009; accepted 11 September 2009; published online 14 October 2009)

\begin{abstract}
Reverse flow is undesirable in liquid crystal devices with vertical alignment. The influence of the material properties on the onset of backflow is investigated for commercially available negative dielectric liquid crystals. It is shown that the threshold voltage $V_{\mathrm{BF}}$ for the occurrence of backflow is an important material characteristic. This threshold is relevant for applications and a large value is desired in devices to avoid backflow while keeping a wide applicable voltage range. Accurate finite element simulation of the liquid crystal hydrodynamics allows extraction of $V_{\mathrm{BF}}$ and the unknown Miesowicz coefficients $\eta_{i j}$. The resulting values are tabulated at $20.0{ }^{\circ} \mathrm{C}$. (C) 2009 American Institute of Physics. [doi:10.1063/1.3242018]
\end{abstract}

The occurrence of reverse nematic flow ${ }^{1}$ in liquid crystal devices with homeotropic alignment ${ }^{2,3}$ has important consequences for practical applications. For instance, vertically aligned liquid crystal displays (VA-LCDs) have become very popular for mobile and television applications because of their excellent contrast ratio and wide viewing angle. However, their dynamic behavior ${ }^{4}$ is affected by the possible occurrence of reverse flow, also referred to as backflow. When a voltage step with amplitude exceeding a certain threshold $V_{\mathrm{BF}}$ is applied, the initial rapid variation causes reverse flow of the negative dielectric liquid crystal which leads to a complex reorientation mechanism (including a twist deformation) and a bounced transient transmission profile, ${ }^{5}$ increasing the switching times. Therefore, the limitations due to backflow must be taken into account as a special case to design improved driving schemes ${ }^{6}$ to achieve fast and reliable switching in VA-LCDs. In this paper, the influence of the material properties on the onset of backflow is investigated for a number of commercially available liquid crystal materials with negative dielectric anisotropy. The viscous flow properties of liquid crystals are described by the four Miesowicz ${ }^{7}$ coefficients $\eta_{i j}$. The three principal coefficients express the viscosity of the liquid crystal for a liquid crystal director parallel to the direction of flow $\left(\eta_{11}\right)$, parallel to the direction of the velocity gradient $\left(\eta_{22}\right)$, and perpendicular to both previous directions $\left(\eta_{33}\right)$, respectively. The fourth coefficient $\eta_{12}$ is related to the shear viscosity. The $\eta_{i j}$ coefficients are, in general, not known because of the notorious difficulty in measuring their values accurately. ${ }^{8}$ It is shown that the threshold voltage $V_{\mathrm{BF}}$ for the occurrence of backflow is a material characteristic comparable to the Freedericksz threshold voltage ${ }^{9}$ for elastic deformation. The backflow threshold is related to the viscous flow properties of liquid crystals and is relevant for practical devices because backflow is undesired and should be avoided. Furthermore, it is possible to estimate the unknown Miesowicz viscosity coefficients $\eta_{i j}$ based on measurement and simulation of $V_{\mathrm{BF}}$. The $\eta_{i j}$ coefficients are extracted using this approach and

${ }^{a)}$ Electronic mail: pieter.vanbrabant@elis.ugent.be. tabulated together with the $V_{\mathrm{BF}}$ values at $20.0{ }^{\circ} \mathrm{C}$ for the nematic mixtures MLC-6609, $-6882,-7026,-6610$, and ZLI-4788 from Merck.

The test cells used in this study are monodomain VA cells with a liquid crystal pretilt of $2^{\circ}$. This pretilt angle is obtained with a competing alignment layer approach ${ }^{10}$ by baking the substrates coated with a mixture of two commercial homeotropic and planar aligning polyamic acids at $185^{\circ} \mathrm{C}$. The samples are assembled with antiparallel rubbing and the cell thickness $d$ is determined accurately from capacitance measurements and interference patterns recorded with a spectrophotometer. Vacuum filling is used to fill the test samples with liquid crystal. The samples are placed between crossed polarizers under a microscope and the switching behavior is studied for the case of direct voltage driving. A temperature control system maintains the temperature at $20.0^{\circ} \mathrm{C}$ for all measurements.

To investigate the flow properties of the liquid crystal, the transient transmission profiles obtained for switching the cells from the black state (no voltage applied) to a voltage in the range of 3-10 V are measured with a photodetector. Figure 1 shows the obtained experimental turn-on times (defined

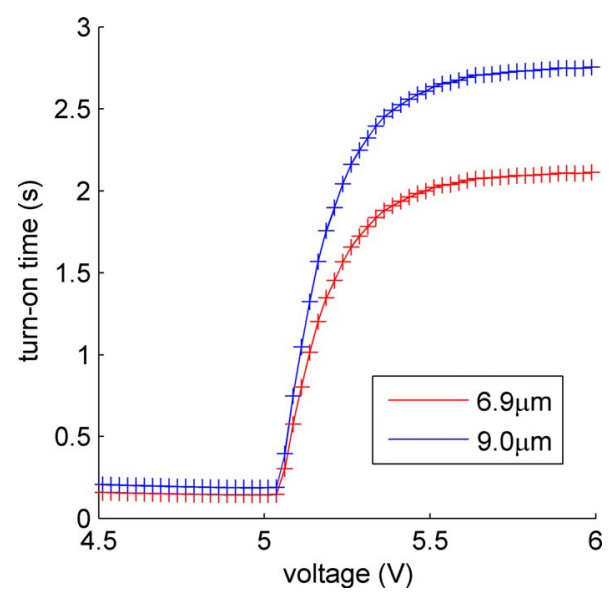

FIG. 1. (Color online) Experimental turn-on times of MLC-6609 as a function of voltage at $20.0{ }^{\circ} \mathrm{C}$ for $d=6.9 \mu \mathrm{m}$ and $d=9.0 \mu \mathrm{m}$. 
as the time required to reach $90 \%$ of the target transmission value starting from the black state) as a function of voltage for two samples filled with the MLC-6609 mixture from Merck with cell thickness of 6.9 and $9 \mu \mathrm{m}$, respectively. Figure 1 shows short turn-on times for low driving voltages (no backflow) whereas the turn-on times are about an order of magnitude longer for voltages exceeding the threshold value $V_{\mathrm{BF}}=5.05 \mathrm{~V}$ due to the occurrence of backflow. Comparing both curves in Fig. 1 shows that the backflow threshold $V_{\mathrm{BF}}$ is independent of the cell thickness. This indicates that the threshold voltage $V_{\mathrm{BF}}$ is a material characteristic comparable to the Freedericksz threshold voltage for elastic deformation.

To model the occurrence of backflow, it is necessary to consider the coupling between the director reorientation and the nematic shear flow. This is described by the classical Leslie-Ericksen continuum theory ${ }^{11,12}$ which gives the equations of motion for nematic liquid crystals with a constant degree of order. The Leslie-Ericksen theory has been generalized more recently by Qian and Sheng ${ }^{13}$ to include changes in the liquid crystal order parameter $S$ to allow for a proper description of disclinations. The viscous flow properties of the liquid crystal material are described in both hydrodynamic theories by the Miesowicz coefficients. A finite element implementation, ${ }^{14}$ in a one-dimensional version, is used to calculate the liquid crystal hydrodynamics of the test cells. Material coefficients for the simulation at $20{ }^{\circ} \mathrm{C}$ are obtained from the available liquid crystal datasheets. It is observed that the simulated threshold voltage $V_{\mathrm{BF}}$ mainly depends on the $\eta_{22}$ coefficient. Therefore, $\eta_{22}$ is considered as an optimization parameter in the simulations. The remaining $\eta_{i j}$ coefficients can be calculated from the value of $\eta_{22}$ as

$$
\begin{aligned}
& \eta_{11}=\gamma-3 \eta_{22}+2 \alpha_{4}+2 \alpha_{6}, \\
& \eta_{33}=\alpha_{4} / 2, \\
& \eta_{12}=\alpha_{1},
\end{aligned}
$$

where $\gamma$ is the (known) rotational viscosity and $\left(\alpha_{1}, \alpha_{4}, \alpha_{6}\right)$ are the Leslie viscosity coefficients ${ }^{11}$ which can be obtained from a recent estimation procedure. ${ }^{15}$ Small variations in boundary conditions at the top and bottom substrates have an important influence on the dynamics of vertically aligned cells. ${ }^{16}$ Therefore, the deviation of the pretilt $(\Delta \theta)$ and the pretwist $(\Delta \phi)$ between the top and bottom substrates has been fitted for one experimental transient transmission profile. This procedure yields $\Delta \theta=1.5^{\circ}$ and $\Delta \phi=2^{\circ}$. Using these boundary conditions, the available datasheet parameters and the extracted set of $\eta_{i j}$ coefficients as input parameters, the dynamic behavior of the test cell at $20^{\circ} \mathrm{C}$ is simulated. The transient optical transmission is calculated from the resulting dynamic liquid crystal director profile with the Jones matrix formalism $^{17}$ for every voltage step. Figure 2 shows the turn-on times as a function of voltage obtained from experiments and simulations for different values of $\eta_{22}$ for the $6.9 \mu \mathrm{m}$ sample filled with MLC-6609.

It can be seen from Fig. 2 that changing the value of $\eta_{22}$ leads indeed to an important shift in the threshold voltage $V_{\mathrm{BF}}$. It is clear that the value $\eta_{22}=15.5 \mathrm{mPa}$ s for MLC-6609 provides excellent agreement in the backflow threshold $\left(V_{\mathrm{BF}}=5.05 \mathrm{~V}\right)$ between experiment and simulation. Furthermore, this value is in agreement with earlier theoretical

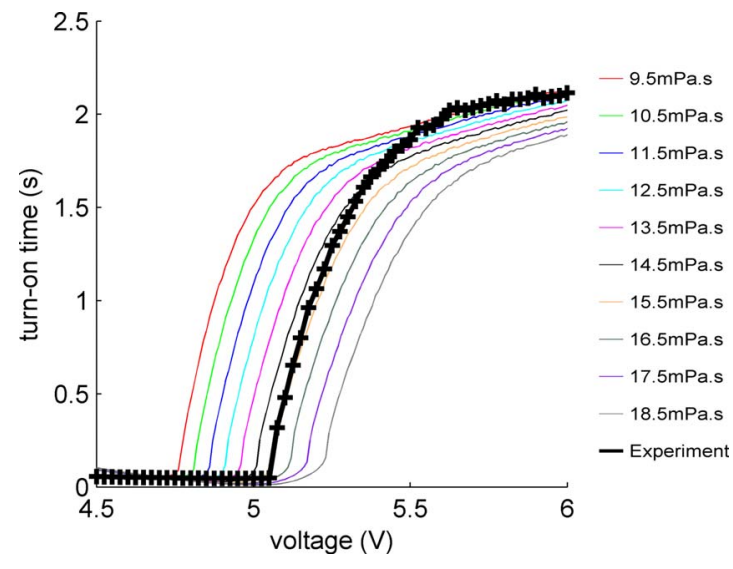

FIG. 2. (Color online) Experimental (bold) and simulated (color) turn-on times of MLC-6609 as a function of voltage at $20.0^{\circ} \mathrm{C}$ for $d=6.9 \mu \mathrm{m}$. The simulated results are obtained for a varying value of $\eta_{22}$ as denoted in the legend.

considerations ${ }^{18}$ on the value of $\eta_{22}$ that yield a lower and upper bound $0.7 \eta_{f}<\eta_{22}<\eta_{f}$, with $\eta_{f}=20 \mathrm{mPa}$ s the dynamic flow viscosity ${ }^{11}$ of MLC-6609. These two observations indicate that the extracted $\eta_{22}$ value and the corresponding set of $\eta_{i j}$ coefficients calculated according to Eqs. (1)-(3) provide a good estimation of the Miesowicz coefficients of MLC-6609 at $20.0{ }^{\circ} \mathrm{C}$. The accuracy on the value of $\eta_{22}$ is estimated as $\pm 0.25 \mathrm{mPa}$ s by considering the relatively large shift of $V_{\mathrm{BF}}$ with a change in $\eta_{22}$, as shown in Fig. 2. Performing an error analysis based on Eqs. (1)-(3) shows that the error on the other $\eta_{i j}$ values is below 5\%. It is observed that a variation of the pretilt and pretwist angles at the substrate interfaces has a negligible influence on the value of $V_{\mathrm{BF}}$ in simulations.

Using the extracted $\eta_{i j}$ coefficients, the dynamic behavior of the test cells has been simulated for a varying cell thickness to check if $V_{\mathrm{BF}}$ remains unchanged, as observed in Fig. 1. The simulated turn-on times of MLC-6609 as a function of voltage in Fig. 3 confirm that the backflow threshold is independent of the cell thickness. This proves that the threshold $V_{\mathrm{BF}}$ is a practical and relevant parameter to describe the viscous flow properties of liquid crystal materials for applications because the applicable voltage range in devices is limited by this threshold to achieve fast and reliable

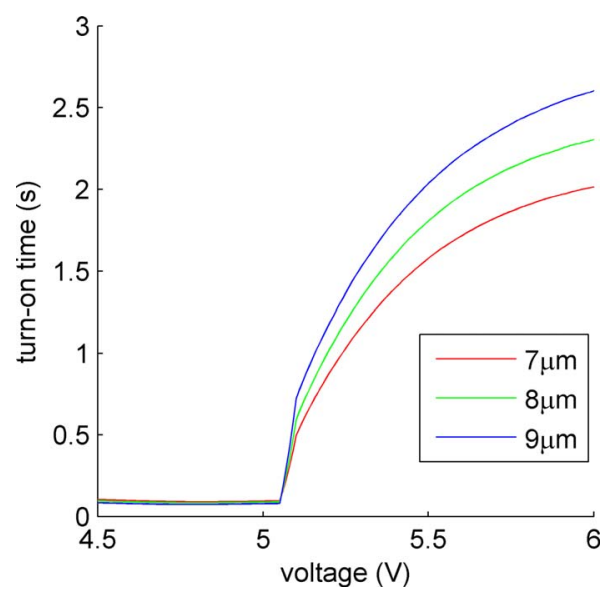

FIG. 3. (Color online) Simulated turn-on times of MLC-6609 as a function of voltage (obtained for $\eta_{22}=15.5 \mathrm{mPa} \mathrm{s}$ ) for different values of the cell thickness $d$. 
TABLE I. Backflow threshold $V_{\mathrm{BF}}$, estimated Miesowicz coefficients $\eta_{i j}$ and rotational viscosity $\gamma$ at $20.0^{\circ} \mathrm{C}$ of MLC-6609, MLC-6610, MLC-6882, MLC-7026-000, and ZLI-4788. The error on the extracted values is calculated as below 5\%.

\begin{tabular}{lccccc}
\hline \hline Material & $\begin{array}{c}V_{\mathrm{BF}} \\
(\mathrm{V})\end{array}$ & $\begin{array}{c}\eta_{11} \\
(\mathrm{mPa} \mathrm{s})\end{array}$ & $\begin{array}{c}\eta_{22} \\
(\mathrm{mPa} \mathrm{s})\end{array}$ & $\begin{array}{c}\eta_{33} \\
(\mathrm{mPa} \mathrm{s})\end{array}$ & $\begin{array}{c}\eta_{12} \\
(\mathrm{mPa} \mathrm{s})\end{array}$ \\
\hline MLC-6609 & 5.05 & 210.3 & 15.5 & 51.8 & -34.2 \\
MLC-6610 & 5.59 & 202.0 & 18.0 & 52.5 & -30.0 \\
MLC-6882 & 4.81 & 188.5 & 14.0 & 53.4 & -25.6 \\
MLC-7026 & 4.66 & 204.0 & 10.0 & 52.5 & -30.0 \\
ZLI-4788 & 4.30 & 378.0 & 13.0 & 52.5 & -30.0 \\
\hline \hline
\end{tabular}

switching. ${ }^{4}$ Knowledge of the Miesowicz coefficients becomes relevant when accurate modeling of the dynamic behavior is desired within the backflow regime $\left(V>V_{\mathrm{BF}}\right)$, but this is not required for most applications. Next to the dependence of $V_{\mathrm{BF}}$ on the Miesowicz coefficients, as illustrated in Fig. 2, this threshold is found to be proportional to the square root of the ratio $K_{33} / \Delta \epsilon$ in analogy with the Freedericksz threshold voltage for elastic deformation in homeotropic cells.

The viscous flow properties of the mixtures MLC-6609, MLC-6610, MLC-6882, MLC-7026-000, and ZLI-4788 from Merck KGaA have been characterized by measurement and simulation of the dynamic characteristics, as presented for MLC-6609 in Figs. 1-3. This allows a comparison of the mixtures in terms of flow performance to select the most suitable material depending on the application. Table I lists the backflow threshold $V_{\mathrm{BF}}$ and Miesowicz coefficients extracted at $20.0^{\circ} \mathrm{C}$, together with the datasheet value of the rotational viscosity $\gamma$. The observed material dependency of the flow properties is reflected in Table I by the variation in the $V_{\mathrm{BF}}$ and $\eta_{i j}$ values for the different mixtures. A large $V_{\mathrm{BF}}$ is desired in practical applications to have a wide applicable voltage range while avoiding the occurrence of backflow. From this point of view, MLC-6610 is preferred over the other mixtures. However, the datasheet value of the rotational viscosity at $20.0{ }^{\circ} \mathrm{C}$ is $\gamma=148 \mathrm{mPa}$, which is relatively high compared to MLC-6882 and MLC-7026-000, leading to higher switching times (also for $V<V_{\mathrm{BF}}$ ). Mixture MLC-6882 has a lower threshold $V_{\mathrm{BF}}=4.81 \mathrm{~V}$ than MLC6610 , but faster switching is obtained due to a lower rotational viscosity $\gamma=108 \mathrm{mPa}$. This illustrates that there can be a trade-off for practical applications between using a faster material at the cost of a lower backflow threshold $V_{\mathrm{BF}}$ or using a slower material with a higher $V_{\mathrm{BF}}$ value. Compared to the other mixtures, ZLI-4788 has the lowest threshold $V_{\mathrm{BF}}=4.30 \mathrm{~V}$ in combination with the highest rotational viscosity $\gamma=309 \mathrm{mPas}$, so this material is undesirable if switching times and backflow effects are important.

In conclusion, the material influence on the occurrence of reverse flow in devices with homeotropic liquid crystal alignment has been investigated. It has been shown that the threshold voltage $V_{\mathrm{BF}}$ for the onset of backflow is a material characteristic. Knowledge of $V_{\mathrm{BF}}$ is important because backflow is undesired in many practical devices. Materials with a high $V_{\mathrm{BF}}$ value are desired for display applications, which leads to an additional factor in the trade-off to select the best mixture for the desired application. Fitting simulations of $V_{\mathrm{BF}}$ with experiments allows one to extract the Miesowicz viscosity coefficients $\eta_{i j}$ of liquid crystals with negative dielectric anisotropy. Using this approach, the values of $V_{\mathrm{BF}}$ and $\eta_{i j}$ have been tabulated at $20.0{ }^{\circ} \mathrm{C}$ for five common nematic mixtures. The variation in these values for the different materials reflects the observed material dependency of the flow properties.

Pieter Vanbrabant is a $\mathrm{PhD}$ Fellow of the Research Foundation-Flanders (FWO Vlaanderen) and Jeroen Beeckman is a Postdoctoral Fellow of the same institution. The work has been carried out in the framework of the Interuniversity Attraction Pole (IAP) project Photonics@be of the Belgian Science Foundation. The authors thank Atsutaka Manabe from Merck Darmstadt for material provision and discussions. Pieter Vanbrabant would like to thank Nathalie Velthoven-Dessaud and Jan Strömer from Philips Research Laboratories, Eindhoven (The Netherlands) for earlier collaboration on backflow in VA-LCDs (Refs. 4 and 6).

${ }^{1}$ P. Pieranski, F. Brochard, and E. Guyon, J. Phys. (Paris) 34, 35 (1973).

${ }^{2}$ M. F. Schiekel and K. Fahrenschon, Appl. Phys. Lett. 19, 391 (1971).

${ }^{3}$ F. J. Kahn, Appl. Phys. Lett. 20, 199 (1972).

${ }^{4}$ P. J. M. Vanbrabant, N. Dessaud, and J. F. Strömer, Appl. Phys. Lett. 92, 091101 (2008).

${ }^{5}$ C. Z. van Doorn, J. Appl. Phys. 46, 3738 (1975).

${ }^{6}$ P. J. M. Vanbrabant, N. Velthoven-Dessaud, J. F. Strömer, and K. Neyts, Appl. Phys. Lett. 93, 261112 (2008).

${ }^{7}$ M. Miesowicz, Nature (London) 158, 27 (1946).

${ }^{8}$ J. W. Summerford, J. R. Boyd, and B. A. Lowry, J. Appl. Phys. 46, 970 (1975).

${ }^{9}$ V. Freedericksz and V. Zolina, Trans. Faraday Soc. 29, 919 (1933).

${ }^{10}$ P. J. M. Vanbrabant, J. Beeckman, and K. Neyts, Liq. Cryst. (to be published), doi:10.1080/02678290903274458.

${ }^{11}$ F. M. Leslie, Q. J. Mech. Appl. Math. 19, 357 (1966).

${ }^{12}$ J. L. Ericksen, Mol. Cryst. Liq. Cryst. 7, 153 (1969).

${ }^{13}$ T. Qian and P. Sheng, Phys. Rev. E 58, 7475 (1998).

${ }^{14}$ R. James, E. Willman, F. A. Fernandez, and S. E. Day, IEEE Trans. Electron Devices 53, 1575 (2006).

${ }^{15}$ H. Wang, T. X. Wu, S. Gauza, J. R. Wu, and S. Wu, Liq. Cryst. 33, 91 (2006).

${ }^{16}$ L. Chen and S. Chen, Jpn. J. Appl. Phys., Part 2 39, 368 (2000).

${ }^{17}$ R. C. Jones, J. Opt. Soc. Am. 46, 126 (1956).

${ }^{18}$ D. Armitage and J. Larimer, SID Int. Symp. Digest Tech. Papers 27, 584 (1996). 